\title{
Le risque intrinsèque à la gestion locale des risques liés à l'eau en Algérie
}

\section{Nadjet Aroua et Ewa Berezowska-Azzag}

\section{CpenEdition}

1 Journals

Édition électronique

URL : http://journals.openedition.org/rge/4628

DOI : $10.4000 /$ rge.4628

ISSN : 2108-6478

Éditeur

Association des géographes de l'Est

Édition imprimée

Date de publication : 31 août 2013

ISSN : 0035-3213

Référence électronique

Nadjet Aroua et Ewa Berezowska-Azzag, « Le risque intrinsèque à la gestion locale des risques liés à l'eau en Algérie », Revue Géographique de l'Est [En ligne], vol. 53 / 1-2 | 2013, mis en ligne le 16

septembre 2013, consulté le 21 décembre 2020. URL : http://journals.openedition.org/rge/4628 ; DOI : https://doi.org/10.4000/rge.4628

Ce document a été généré automatiquement le 21 décembre 2020.

Tous droits réservés 


\title{
Le risque intrinsèque à la gestion locale des risques liés à l'eau en Algérie
}

\author{
Nadjet Aroua et Ewa Berezowska-Azzag
}

\section{INTRODUCTION}

1 En Algérie, en l'espace de quelques mois d'intervalle, la presse nationale peut rapporter des titres aussi contradictoires que les événements hydroclimatiques auxquels ils sont liés. Déjà en 2004, on y lisait l'annonce par le Ministère des ressources en eau d'une baisse de dotation à partir de l'année suivante puis le lancement de nombreux barrages à travers les différentes régions du pays. Mi-2005, au moins treize wilayas (la wilaya est l'équivalent du Département en France) affichaient les signes d'une sécheresse hydraulique manifeste accélérant l'adoption de huit décrets exécutifs au bénéfice d'opérations hydrauliques urgentes dites d'intérêt public. La même année étaient relevées de graves insuffisances dans l'assainissement urbain et la solution du dessalement d'eau de mer se voyait octroyer un budget de plusieurs milliards de dollars. 


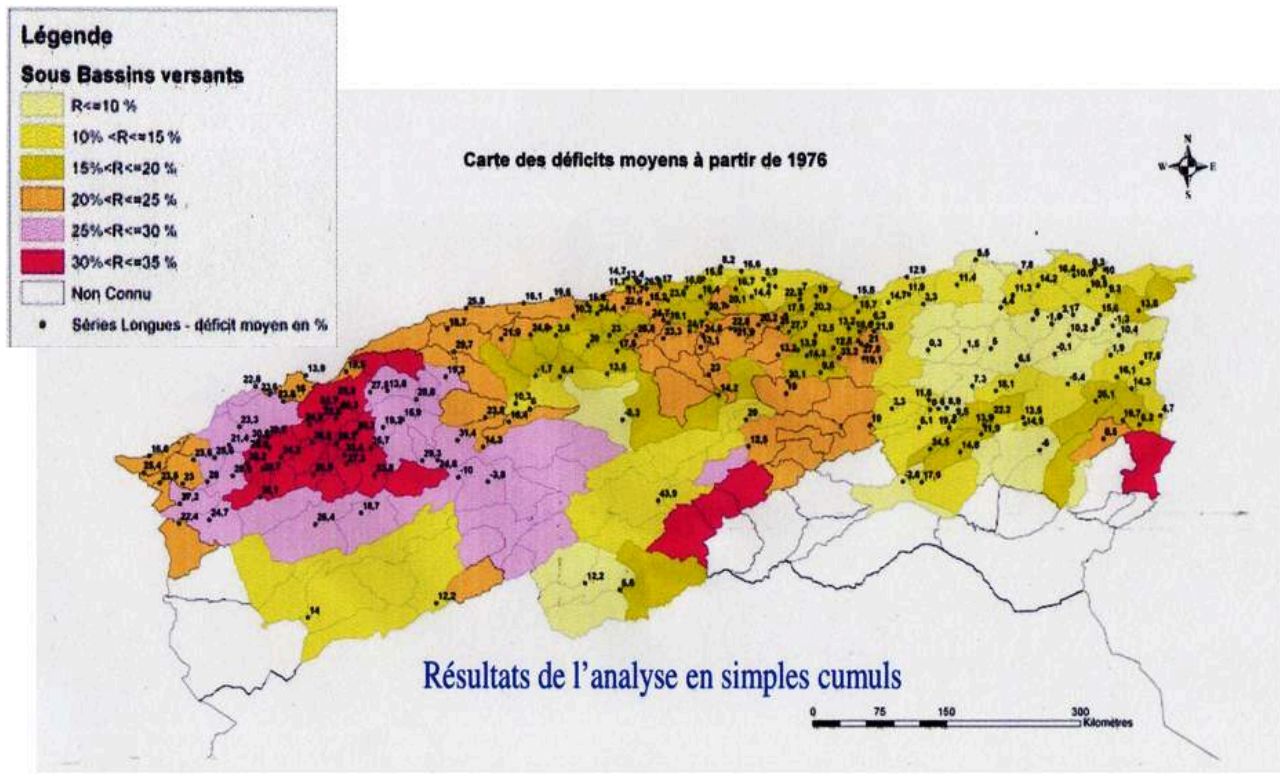

Carte 1 : Déficit pluviométrique en Algérie entre 1976 et 2010 (Fond Agence Nationale des Ressources Hydriques- ANRH, 2010)

2 La persistance de la sécheresse, notamment entre 1980 et 2000, avait conduit l'Algérie à l'élaboration d'un Plan National de l'Eau (PNE) basé sur une déficience chronique de la ressource (Ministère de l'Aménagement du Territoire et de l'Environnement, 2001). Dans la conjoncture de stress hydrique, la production avait en effet connu une réduction notoire justifiant le recours aux ressources non conventionnelles par dessalement d'eau de mer ou réutilisation d'eaux usées épurées. Ainsi la demande en eau avait-elle été sensiblement améliorée dans les grandes villes, mais non complètement satisfaite (Aroua 2005).

La sécheresse avait en effet tourmenté les esprits jusqu'en 2006 avant de céder la place (en 2007) à un automne exceptionnellement pluvieux. Début 2008, de nouveau la sécheresse menaçait l'Algérie puis l'année s'achevait sur le même scénario de crues et inondations, notamment dans les régions Ouest et Sud-ouest, qui s'est prolongé jusqu'à l'hiver suivant (www.meteo.dz). Les événements pluviométriques remarquables qui ont provoqué des débordements ou crues d'oueds, des inondations et glissements de terrains ont occasionné de lourdes pertes humaines et matérielles. Suite à l'effondrement ou grave endommagement de leurs habitations, le nombre de familles sinistrées était chaque fois sans commune mesure avec celui des victimes car les régions enclavées n'avaient pu être secourues rapidement, la communication ayant été rompue (fermeture des routes, coupures d'électricité et de lignes téléphoniques). 


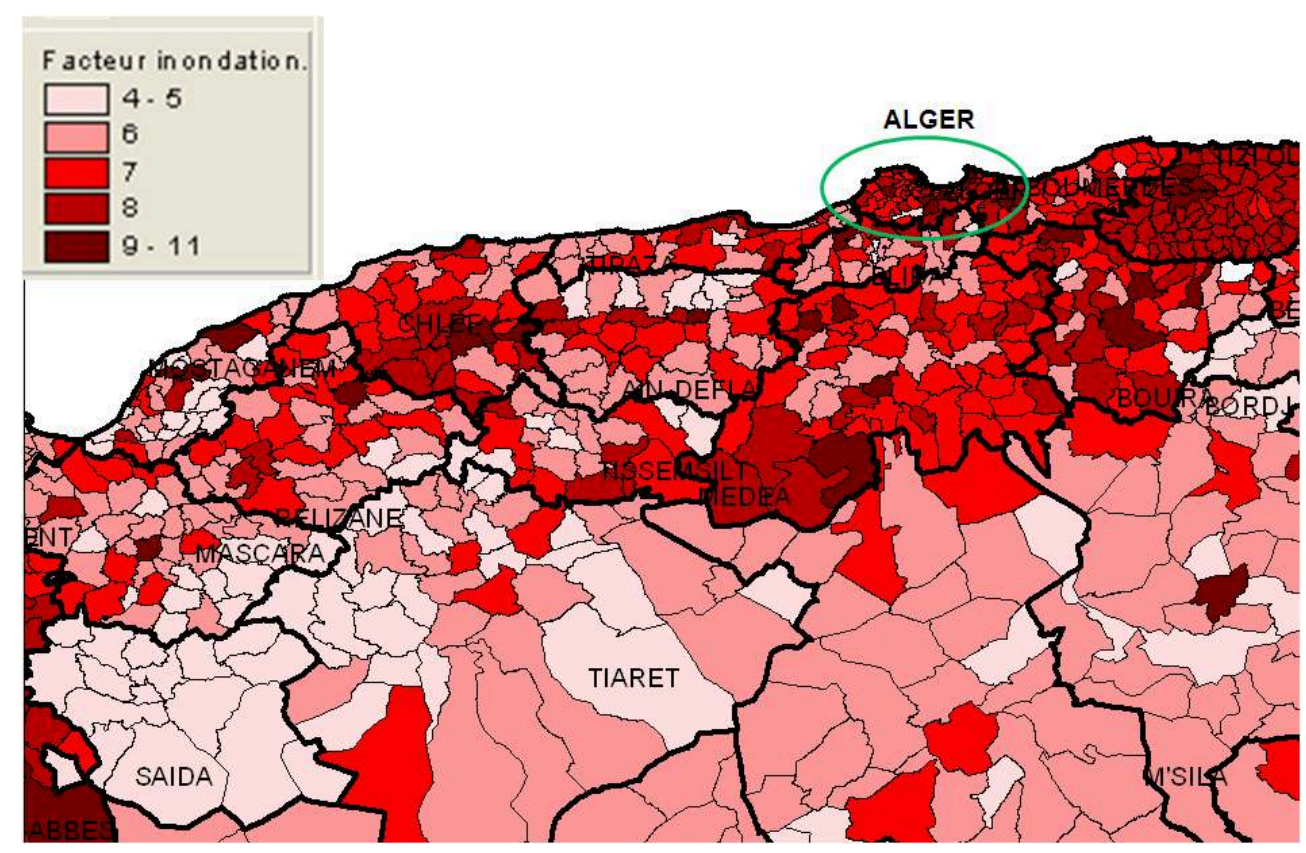

Carte 2 : Distribution du facteur inondation en Algérie (L. BAHLOULI, non daté)

4 Chaque année presque toutes les wilayas subissent impuissantes les conséquences tragiques des inondations ou de la sécheresse. Mais, alors que les autorités continuent à plaider le caractère naturel quoiqu'exceptionnel de tels événements, plusieurs localités à travers le pays tentent de faire face aux nombreux dégâts occasionnés avec les moyens de bord, c'est-à-dire dans le dénuement, parfois sans plan de secours préétabli (Aroua 2012). Les populations excédées portent alors leur colère et leur peur devant le siège des autorités locales dénonçant leur lenteur et manque d'efficacité. Et celles-ci de répliquer tantôt en accusant le vieux bâti et l'habitat précaire, tantôt en relativisant l'événement et le ramenant à un problème planétaire de changement climatique.

5 Est-ce un problème de politique de développement? De gestion urbaine? De moyens d'action? A l'issue de cette réflexion, il peut être argué qu'il s'agit de ces trois paramètres à la fois. Car, la stratégie nationale de prévention des risques naturels qui semble cibler leurs effets, serait insuffisante en la matière. Or, si les objectifs à long terme n'expriment pas cette ambition, alors les interventions locales ne peuvent que se perdre dans les méandres des échelles spatiotemporelles et des programmes d'action improvisés sous la pression de l'urgence. Pour autant que la gestion locale du risque consiste à contrôler les événements dérivés des phénomènes hydroclimatiques, l'analyse multicritère des facteurs stratégiques, réglementaires et opérationnels qui la déterminent, permettrait d'identifier certaines insuffisances structurelles dont l'impact à terme cultive le risque au lieu de le réduire. L'objectif du présent article est de tester cette hypothèse dans le cas de l'Algérie.

\section{METHODE ET OUTILS.}

6 Sur le plan stratégique, la loi nº4-20 du 25/12/2004 relative à la prévention des risques majeurs et à la gestion des catastrophes dans le cadre du développement durable, fait principalement référence aux Codes de l'eau, la santé, l'urbanisme et l'aménagement du territoire. Elle se fixe comme principal objectif d'édicter les règles de prévention des 
risques majeurs (Art.1) à travers la limitation de la vulnérabilité humaine et matérielle (Art.3) et la mise en œuvre d'un système d'intervention spécifique à l'aléa en cause (Art. 4). A ce titre, elle institutionnalise un plan de prévention adopté par décret (Art.16) doublement spécifique par rapport au risque majeur d'une part et au périmètre géographique d'autre part. Ainsi si les systèmes de veille et d'alerte qu'il détermine sont transposables à d'autres contextes, les mesures d'atténuation sont-elles nécessairement spécifiques à un niveau et un type de vulnérabilité donnée (Art.17).

Selon le Décret n85-232 du 25/08/1985 relatif à la prévention des risques de catastrophes, (la loi n ${ }^{\circ} 04-20$ n'ayant pas été encore suivie de textes réglementaires à ce jour, c'est le décret 85-232 qui régit la prévention des risques de catastrophe), le Wali (équivalent du Préfet en France) est chargé de la mise en œuvre des mesures de prévention des risques naturels et/ou technologiques (Art.3). En outre, c'est le Président de la commune qui est chargé de leur exécution locale afin de garantir la sécurité des personnes et des biens et la salubrité publique (Art. 69). En cas de danger grave et imminent, il peut prescrire les mesures de sûreté nécessaires avant d'en informer le Wali (Art. 71). Au début de chaque année, sur la base d'un rapport du service technique ou de l'organisme concerné, le Wali et les maires conviennent d'un dispositif de secours déclinant un plan ORSEC spécifique à chaque aléa ainsi que les intervenants tenus de veiller à son exécution parmi les services de sûreté, de gendarmerie et l'ensemble des organes administratifs locaux.

C'est au service d'hydraulique de la Wilaya que revient le soin d'élaborer un plan de prévention des inondations et risques climatiques de forte pluviométrie, éventuellement de les adapter au contexte communal (Décret nº5-232 du 25/08/1985 relatif à la prévention des risques de catastrophes, Art.3). La commune est alors chargée de veiller à son exécution avec l'aide du service de protection civile (Code communal $n^{\circ}$ 90-08 du 04/04/1990, Art.69). En temps de crise suite à une longue sécheresse, la commune s'attache aux mesures d'urgence prescrites par le Plan ORSEC, tandis que le programme de distribution est établi par la société de gestion de l'eau potable. Le programme de mobilisation est établi par le Ministère des ressources en eau et mis en œuvre par l'Agence nationale des barrages ou la Direction de l'Hydraulique de la Wilaya pour ce qui relève de l'exploitation des ressources souterraines. Au titre de ses prérogatives (La loi n ${ }^{\circ} 90-08 \mathrm{du}$ 07/12/1990 fixe les attributions de la commune pour ce qui concerne l'urbanisme, l'habitat, l'infrastructure, le développement économique, les équipements publics, l'hygiène, la salubrité et l'environnement), la commune s'appuie sur les services administratifs et techniques internes et notamment celui de l'urbanisme et le bureau d'hygiène communale.

D'autre part, la réglementation spécifique aux aléas hydroclimatiques relève directement des outils juridiques relatifs à l'eau. Or, bien que prévus par la loi 04-20 relative à la prévention des risques majeurs, les plans de prévention des risques ne font pas encore l'objet de texte réglementant les modalités de leur élaboration. Par ailleurs, tous les bassins hydrographiques n'ayant pas encore fait l'objet d'un schéma d'aménagement des ressources en eau (SAGE, institué par le Code de l'eau) par l'Agence du Bassin Hydrographique dont ils relèvent, la gestion intégrée des ressources en eau se limite pour l'heure aux opérations d'alimentation en eau potable et assainissement, commandées par les plans de gestion établis par la société de gestion des eaux et de l'assainissement de la Wilaya. Celle-ci s'efforce de répondre aux besoins urgents en multipliant le volume de ressources mobilisées aux dépens de la balance hydraulique et 
malgré le nombre important de décrets qui subordonnent la prévention des risques liés à l'eau.

Tableau 1 : Outils réglementaires de prévention des risques liés à l'eau en Algérie.

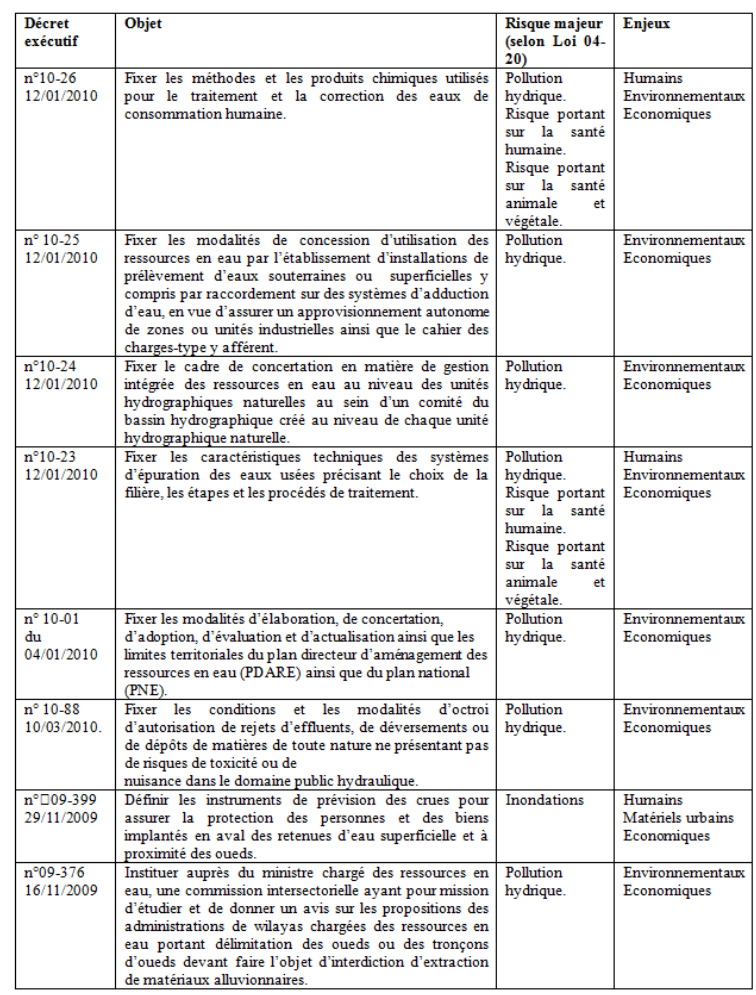

Le Ministère de l'Intérieur et des Collectivités locales prévoit l'élaboration d'un Plan communal d'intervention en cas de risque majeur dont l'objectif serait d'aider la commune à faire face à un évènement extrême. Tel que défini, le dispositif opérationnel, bien que réactif, se voudrait néanmoins anticipatif. En ce sens où les mesures qu'il introduit seraient non seulement adaptées à l'événement et au périmètre considérés mais également applicables aux différentes phases de l'aléa. Préventives avant le sinistre, elles viseraient l'évaluation du risque et la réduction de la vulnérabilité relevant de l'aménagement du territoire, des techniques de construction et de la préservation des systèmes écologiques. Organisationnelles et immédiates au moment et après le sinistre, elles viseraient la limitation des effets négatifs de l'aléa à travers un programme de remise en état (MICL, 2011).

11 Concernant les inondations, des prescriptions particulières seraient déduites de la carte nationale d'inondabilité, déjà établie par l'ANRH, de la hauteur d'eau de référence servant à la délimitation des zones de servitude et donc à la réglementation de l'usage du sol, des travaux d'aménagement à prévoir ainsi que des seuils d'alerte (Art.24). La forte pluviométrie et la sécheresse étant citées parmi les aléas climatiques, le plan de prévention détermine de même les zones exposées et les modalités de veille (Art.27) et étend le champ de prévention « à toute mesure applicable » (Art.28).

12 Selon la loi 04-20 précitée, le dispositif de sécurité face aux risques liés à l'eau veille principalement sur les équipements stratégiques tels que les centres de commandement, les équipements de santé, les liaisons routières et de télécommunication, les bâtiments à caractère patrimonial, qui doivent faire l'objet 
d'expertise régulière, de moyens adéquats et de plans de confortement prioritaires. Par souci d'efficacité, la loi prévoit également un système national d'indemnisation et de recours à la procédure d'expropriation pour cause d'utilité publique. Les mesures structurelles appuient quant à elles la gestion du Plan ORSEC spécifique à un aléa et un écosystème urbain donnés (Art.52). Il est utile de prendre note ici de sa rigueur en termes de phasage et d'organisation des opérations d'une part, ainsi que des moyens humains et matériels envisagés d'autre part (Art.53).

13 A titre d'exemple, le Plan ORSEC-sécheresse, fait référence au Rapport-sécheresse dressé par le service d'hydraulique de la Wilaya suite à un déficit pluviométrique sur la base d'un constat de réduction du volume d'eau stocké dans les barrages, de la production d'eau souterraine due au rabattement du niveau de la nappe et des objectifs de satisfaction de la demande en eau potable suivant le programme de distribution établi par l'organe chargé de la gestion de l'eau. Le Plan ORSEC-sécheresse, communiqué aux communes par le Wali sous forme d'Arrêté, restreint alors l'usage de l'eau potable et interdit le branchement de suppresseur sur le réseau public.

De même, les risques sanitaires liés à l'eau font-ils l'objet d'une circulaire interministérielle communiquée aux walis. La prévention des maladies à transmission hydrique (MTH), dont la commission nationale dépend directement du MICL, définit également un programme d'actions prioritaires sur la base d'un constat épidémiologique établi à la faveur des missions d'inspection d'un comité interministériel et dont les intervenants sont le secteur de l'hydraulique, la santé et les communes. Au niveau de la commune, c'est le Bureau d'Hygiène Communale qui dresse les statistiques des MTH et les transmet à l'Institut National de Santé Publique (INSP) via le Service d'épidémiologie et de médecine préventive (SEMEP).

Si la gestion locale des risques liés à l'eau consiste à s'adapter à une menace et à atténuer ou éviter le danger qu'ils peuvent présenter, elle dépend de facteurs structurels d'ordre stratégique, réglementaire et opérationnel qui la caractérisent (Aroua 2012). Néanmoins il est nécessaire de distinguer la capacité d'adaptation - ou adaptabilité - de la capacité à faire face. Cette dernière représente l'aptitude locale d'absorption du choc, d'adaptation immédiate, autonome, à court terme, aux effets de la catastrophe pendant sa phase d'occurrence : programme d'urgence, organisation des secours, évacuation des victimes, aide aux sinistrés, etc. (Adger 2006) Tandis que la capacité d'adaptation permet de s'adapter à un changement ou une perturbation, notamment climatique, à atténuer les pertes potentielles et assumer leurs impacts (Brooks 2005).

Dans le contexte hydroclimatique de l'Algérie, la gestion des risques liés à l'eau demeure étroitement liée à un ensemble d'indicateurs dont la pertinence est confirmée par des éléments de diagnostic et de preuve tangibles (Aroua 2012). Ils font ici l'objet d'une analyse multicritère suivant deux valeurs d'appréciation: la difficulté d'application locale d'une part et leur degré d'implication dans la capacité d'adaptation ou résilience urbaine d'autre part. En définitive, en relation avec les critères institutionnels, réglementaires et opérationnels, neuf (09) indicateurs pertinents par rapport à la problématique étudiée peuvent être retenus et déclinés comme suit: 
Tableau 2 : Facteurs structurels de gestion des risques liés à l'eau.

\begin{tabular}{|c|c|c|c|c|c|c|}
\hline \multirow{3}{*}{$\begin{array}{l}\text { INDICATEURS } \\
\begin{array}{l}\text { Système de } \\
\text { prévention et de } \\
\text { protection }\end{array}\end{array}$} & \multicolumn{6}{|c|}{$\begin{array}{c}\text { FACTEURS STRUCTURELS } \\
\text { DE CONTROLE DES RISQUES LIES A L'EAU }\end{array}$} \\
\hline & \multicolumn{2}{|c|}{$\begin{array}{l}\text { STRATEGIQUES- } \\
\text { INSTITUTIONNELS }\end{array}$} & \multicolumn{2}{|c|}{ REGLEMENTAIRES } & \multicolumn{2}{|c|}{ OPERATIONNELS } \\
\hline & IAS1 & $\begin{array}{l}\text { Références } \\
\text { juridiques de la } \\
\text { prévention des } \\
\text { AHCU (Loi } \\
\text { Risques } \\
\text { majeurs, Eau, } \\
\text { Santé, } \\
\text { urbanisme, } \\
\text { énergie, } \\
\text { aménagement } \\
\text { territoire et } \\
\text { DD) }\end{array}$ & IAR1 & $\begin{array}{l}\text { Plan de gestion } \\
\text { des ressources } \\
\text { hydriques } \\
\text { (SAGE), Plan de } \\
\text { prévention des } \\
\text { AHCU (PPR), } \\
\text { Plan } \\
\text { d'aménagement } \\
\text { urbain (POS, PU) }\end{array}$ & IAO1 & $\begin{array}{l}\text { Plan ORSEC-AHCU, } \\
\text { (moyens humains et } \\
\text { matériels, Relais } \\
\text { communautaires/ONG, note } \\
\text { et circulaire } \\
\text { interministérielles/dispositif } \\
\text { de prévention et de lutte } \\
\text { contre les AHCU) }\end{array}$ \\
\hline $\begin{array}{l}\text { Système de } \\
\text { prévision, alerte } \\
\text { et secours }\end{array}$ & IAS2 & $\begin{array}{l}\text { Système } \\
\text { d'acteurs et } \\
\text { processus de } \\
\text { décision (Code } \\
\text { Wilaya, Code } \\
\text { commune) }\end{array}$ & IAR2 & $\begin{array}{l}\text { Etude de danger, } \\
\text { Etude d'impact, }\end{array}$ & IAO2 & $\begin{array}{l}\text { Arrêté catastrophe, Arrêté } \\
\text { Zone sinistrée, Décision } \\
\text { d'intervention }\end{array}$ \\
\hline $\begin{array}{l}\text { Capacités } \\
\text { locales } \\
\text { d'application }\end{array}$ & IAS3 & $\begin{array}{l}\text { Prérogatives et } \\
\text { organes } \\
\text { communaux de } \\
\text { prévention des } \\
\text { AHCU, }\end{array}$ & IAR3 & $\begin{array}{l}\text { Plan } \\
\text { d'organisation } \\
\text { des interventions } \\
\text { et secours, } \\
\text { Prévision des } \\
\text { crues, } \\
\text { (communication, } \\
\text { coordination, } \\
\text { information) }\end{array}$ & IAO3 & $\begin{array}{l}\text { Organes de secours, } \\
\text { sauvetage, soins, évacuation, } \\
\text { hygiène (localisation, } \\
\text { moyens humains et } \\
\text { matériels) }\end{array}$ \\
\hline
\end{tabular}

17 La capacité de contrôle des risques liés à l'eau dépend par conséquent de la capacité de gestion, de l'accès aux ressources financières et technologiques et à l'information, de l'existence d'infrastructures et de l'environnement institutionnel et politique (Brooks 2005). Elle est spécifique à un pays, une communauté, un groupe social ou un individu. Elle varie en valeur et en nature à travers le temps et l'espace. (Alwang 2001). Selon cette hypothèse, il peut être argué que la somme arithmétique des scores obtenus par les critères structurels cités contribue à déterminer le niveau de gestion locale des risques liés à l'eau en lui attribuant un indice de qualité. Dans cette logique, ce dernier serait égal à la moyenne des indices obtenus par ces mêmes critères. D'évidence, un indicateur inopérationnel ou temporairement opérationnel ne fournit pas de bénéfice réel à la commune et par conséquent ne peut contribuer à sa résilience dont l'horizon est stratégique par définition.

Tableau 3 : Barème de notation et classement des indicateurs de Résilience.

\begin{tabular}{|c|c|c|c|c|c|c|}
\hline Note & $\begin{array}{l}\text { Niveau d'application } \\
\text { locale }\end{array}$ & $\begin{array}{l}\text { Implication dans la } \\
\text { résilience locale }\end{array}$ & $\sum$ & Indice & \multicolumn{2}{|c|}{$\begin{array}{l}\text { Niveau d'impact } \\
\text { de l'indicateur }\end{array}$} \\
\hline 1 & $\begin{array}{l}\text { Non opérationnel } \\
\text { localement }\end{array}$ & Négligeable à nulle, & 2 à 3 & Jusqu'à 0,37 & Mineur & 1 \\
\hline 2 & $\begin{array}{l}\text { Opérationnel dans } \\
\text { l'urgence }\end{array}$ & Notable à CT & 4 à 5 & $0,37>$ indice $\leq 0.62$ & Notable & 2 \\
\hline 3 & $\begin{array}{l}\text { Opérationnel sur le } \\
\text { plan de la protection }\end{array}$ & Forte à $\mathrm{C}$ et $\mathrm{MT}$ & 6 à 7 & 0,62 >indice $\leq 0.87$ & Critique & 3 \\
\hline 4 & $\begin{array}{l}\text { Opérationnel sur le } \\
\text { plan de la prévention }\end{array}$ & $\begin{array}{l}\text { Très forte à } \mathrm{C}, \mathrm{M} \text { et } \\
\text { LT }\end{array}$ & 8 & 0,87 >indice $\leq 01.00$ & Majeur & 4 \\
\hline
\end{tabular}

18 Leur caractérisation montre que les facteurs structurels de gestion des risques liés à l'eau obtiennent un score de $27 / 72$. 
Tableau 4 : Caractérisation des facteurs et indicateurs structurels d'Adaptation locale.

\begin{tabular}{|c|c|c|c|c|c|c|c|c|c|}
\hline $\begin{array}{l}\text { FACTEURS } \\
\text { STRUCTURELS } \\
\text { D'ADAPTATION }\end{array}$ & \multicolumn{3}{|c|}{$\begin{array}{l}\text { FACTEURS } \\
\text { STRATEGIQUES- } \\
\text { INSTITUTIONNELS }\end{array}$} & \multicolumn{3}{|c|}{$\begin{array}{l}\text { FACTEURS } \\
\text { REGLEMENTAIRES }\end{array}$} & \multicolumn{3}{|c|}{$\begin{array}{l}\text { FACTEURS } \\
\text { OPERATIONNELS }\end{array}$} \\
\hline Indicateurs & IAS1 & IAS2 & IAS3 & IAR1 & IAR12 & IAR3 & IAO1 & IAO2 & IAO3 \\
\hline $\begin{array}{l}\text { Note indicateur }(/ 4) \\
\text { / Niveau d'application } \\
\text { locale }\end{array}$ & $1 / 4$ & $2 / 4$ & $2 / 4$ & $1 / 4$ & $1 / 4$ & $2 / 4$ & $2 / 4$ & $1 / 4$ & $2 / 4$ \\
\hline $\begin{array}{l}\text { Note indicateur (/4) } \\
\text { implication dans la } \\
\text { résilience urbaine }\end{array}$ & $1 / 4$ & $2 / 4$ & $2 / 4$ & $1 / 4$ & $1 / 4$ & $2 / 4$ & $2 / 4$ & $1 / 4$ & $1 / 4$ \\
\hline $\begin{array}{l}\text { Note globale indicateur } \\
\text { (/8) }\end{array}$ & $2 / 8$ & $4 / 8$ & $4 / 8$ & $2 / 8$ & $2 / 8$ & $4 / 8$ & $4 / 8$ & $2 / 8$ & $3 / 8$ \\
\hline Indice spécifique & 0.25 & 0.50 & 0.50 & 0.25 & 0.25 & 0.50 & 0.50 & 0.25 & 0.37 \\
\hline $\begin{array}{l}\text { Note globale facteur } \\
(/ 24)^{*}\end{array}$ & \multicolumn{3}{|c|}{10} & \multicolumn{3}{|c|}{8} & \multicolumn{3}{|c|}{9} \\
\hline $\begin{array}{l}\text { Indice synthétique (du } \\
\text { facteur) }\end{array}$ & \multicolumn{3}{|c|}{0.41} & \multicolumn{3}{|c|}{0.33} & \multicolumn{3}{|c|}{0.37} \\
\hline $\begin{array}{l}\text { Note de gestion locale } \\
(/ 72)\end{array}$ & \multicolumn{9}{|c|}{27} \\
\hline Indice d'Adaptation IA & \multicolumn{9}{|c|}{0.37} \\
\hline
\end{tabular}

19 Leur performance temporaire serait basée sur l'organisation du système de secours élaboré à l'échelle wilayale, bien plus que celui de prévision. Bien que désignant la capacité de maîtrise des aléas hydroclimatiques, en vérité ils échappent à l'autorité communale qui tient le rôle d'exécutant des plans d'intervention pendant la survenance de l'aléa. Certes le Bureau d'hygiène communale assure un contrôle régulier de la qualité de l'eau, la collecte des déchets divers, l'assainissement et les maladies infectieuses. Or, comme signalé précédemment, il s'agit-là d'une opération préventive malheureusement sans grand impact sur la salubrité des communes dont les moyens et l'autorité demeurent généralement en deçà des besoins en matière d'hygiène et de sécurité.

Les quatre indicateurs (IAS2, IAS3, IAR3 et IAO1) qui obtiennent la moitié de la note $(4 / 8$ pts) relèvent respectivement de facteurs stratégiques, réglementaires et opérationnels et doivent ce relatif avantage à leur opérationnalité dans l'urgence autant qu'à leur notable implication à court terme dans la résilience locale. De fait, la division réglementaire des tâches entre les acteurs (IAS2 et IAS3) ainsi que l'établissement des plans de secours (ORSEC) qu'ils désignent, garantissent à la commune le secours mais non la sécurité. Le faible niveau de gestion locale s'expliquerait par ailleurs par l'inapplication de quatre (04) indicateurs (IAS1, IAR1, IAR2 et IAO2) très probablement liée à l'absence d'outils réglementaires d'une part, et la grande difficulté d'application de cinq (05) indicateurs de type stratégique et organisationnel d'autre part (IAS2, IAS2, IAR3, IAO1, et IAO3). Ce qui prête à croire que la gestion locale des risques liés à l'eau ne cible ni la réduction de leur effets par des mesures de protection, ni l'adaptation préventive.

21 A ce titre, il semble utile de signaler que le niveau d'application qui affecte certains indicateurs structurels de gestion serait lié à plusieurs facteurs. L'absence d'outils réglementaires et de moyens matériels et financiers n'explique que partiellement la faible qualité de gestion locale. Si le niveau de formation du personnel des services techniques communaux n'est pas mis en doute, il nécessite néanmoins une mise à niveau suivant les nouveaux enjeux et pratiques de gouvernance. D'autre part, seuls les facteurs opérationnels (IAO1 et IAO2) semblent à juste titre opérationnels et pour cause ils désignent des procédures d'urgence. En l'absence de stratégie et de plans, les communes ne peuvent prétendre contrôler les aléas hydroclimatiques ni les prévenir. 
Certes les mécanismes du système d'acteurs (IAS2 et IAS3) sont définis juridiquement, mais ils semblent handicapés par l'étroite dépendance communale vis-à-vis de l'autorité supérieure et ne fonctionner qu'en cas d'urgence.

En définitive, la note des indicateurs de gestion des risques ne se détache des axes du repère que soutenue par les plans de secours d'urgence et l'aide extérieure concédée par la Wilaya. La gestion actuelle ne couvre que les court et moyen termes, alors qu'elle est supposée orienter la stratégie de développement vers des alternatives d'avantgarde servant à la fois les objectifs de santé, de sécurité et de durabilité, en l'occurrence, les indicateurs liés à la stratégie du système d'acteurs (IAS2) et aux prérogatives de la commune (IAS3), à l'organisation réglementaire des secours (IAR3) et la procédure opérationnelle de plan ORSEC (IAO1). Cinq indicateurs sur neuf semblent faillir, essentiellement liés aux facteurs politiques et économiques. Manifestement une règlementation non cautionnée par une politique volontariste et un système économique stable, ne permet pas d'élever le niveau de sécurité locale face aux risques liés à l'eau.

L'approche participative nécessaire à la mise en œuvre efficace de ces mesures et dispositifs, préconisée par le Ministère de l'Intérieur et des collectivités locales et celui de l'aménagement du territoire et de l'environnement demeure quant à elle lettre morte malgré le recensement de nombreuses associations civiles au niveau national (MICL, 2011). La portée de l'ensemble de ces indicateurs pourrait être améliorée grâce à l'investissement à long terme des potentialités humaines, environnementales et économiques. La généralisation de certaines procédures telles que la concertation interbassin, l'intersectorialité et l'intercommunalité et la participation civile pourrait contribuer à l'amélioration du niveau de résilience à moyen et long termes.

\section{RESULTATS ET INTERPRETATION}

En Algérie, les catastrophes dites naturelles enregistrées à travers le pays ont révélé sa grande vulnérabilité. A ce jour notre pays leur paie un lourd tribut en termes de vies humaines, sinistres, dégâts environnementaux, économiques et matériels. Chaque événement, même mineur, non seulement retarde le processus de développement, mais greffe son bilan en détruisant certains acquis fondamentaux fonctionnels, structurels et relationnels. Certes un dispositif législatif lié aux risques majeurs existe. Il repose essentiellement sur la loi $n^{\circ}$ 04-20 du 25 décembre 2004 relative à la prévention des risques majeurs et à la gestion des catastrophes dans le cadre du développement durable. Loi, dont un récent rapport du Ministère de l'intérieur et des collectivités locales (MICL), regrette l'inefficacité en l'absence de textes d'application réglementant sa mise en œuvre, ainsi que les nécessaires mécanismes de coordination intersectorielle qui la soutiennent (Ouldamer 2008). Hormis la procédure de plan ORSEC, aujourd'hui aucun processus administratif ou système d'acteurs ne permet de prévenir efficacement le risque de catastrophe (Fernini 2008).

De plus, la principale référence juridique de prévention des risques majeurs (Loi n $04-20$ du 25/12/2004) bien qu'ayant pour objectif l'atténuation de la vulnérabilité (Art. 18), ne semble pas investir l'approche systémique. Pas plus qu'elle ne précise le seuil de risque au-delà duquel un aléa se transforme en catastrophe majeure, si ce n'est l'évocation non quantifiée de «dommages au plan humain, social, économique et/ou environnemental » (Art. 4). Il peut être remarqué également une certaine confusion des 
aléas, de l'événement initiateur externe (climatique) et initial (hydrographique) d'une part et des aléas dérivés qui en résultent (événements terminaux hydroclimatiques urbains) d'autre part. A ce titre, les inondations qui sont des aléas urbains dérivés d'aléas hydroclimatiques, en l'occurrence crue d'oued ou remontée de nappe, seraient improprement citées parmi les aléas naturels à l'exemple des séismes et des variations climatiques. Il semblerait cependant que même l'OMS et l'ONU les classent parmi les aléas naturels. De même des pollutions hydriques, les feux de forêts ou risques portant sur la santé humaine qui ne peuvent être que conséquents et non mis en cause et dont l'origine peut être un aléa naturel (par exemple climatique) ou anthropique (par exemple technologique).

En l'attente de textes réglementaires qui pourraient lever la confusion, il peut être d'ores et déjà noté, selon le même texte de loi, que le plan général de prévention des risques tel qu'institué, devrait contenir trois types de documents (Art. 18):

les références méthodologiques servant à l'évaluation de l'aléa, probablement en termes de gravité et de fréquence, c'est-à-dire d'impact potentiel, les documents cartographiques de localisation des zones vulnérables, enfin, les mesures de prévention et d'atténuation de la vulnérabilité " en matière d'établissement humains et d'occupation de l'espace» déduites des résultats précédents c'est-à-dire de l'évaluation de l'aléa et du niveau de vulnérabilité. Il est nécessaire de reconnaitre à ce plan la méthodologie scientifique d'appréciation du risque potentiel, désormais référencée et basée sur des savoirs théoriques et empiriques relatifs à un aléa donné. Appelé à fournir non seulement le zonage mais également les servitudes réglementaires régissant l'occupation de l'espace dans les sites vulnérables, il se met directement au service de l'aménagement du territoire et de l'urbanisme. Il serait cependant utile d'étudier également les sites dont la localisation ou les activités pourraient contribuer à la propagation des dégâts, quand bien même ils ne seraient pas directement exposés. Autrement dit, se pencher non seulement sur les enjeux majeurs tels qu'infrastructure routière, équipements de soins et de commande etc., mais aussi sur les établissements classés et zones d'habitations précaires.

En termes de nuisance, le potentiel destructif de la stagnation d'eaux pluviales pourrait sembler négligeable. Sa durée d'impact, de quelques jours, pourrait néanmoins provoquer l'endommagement de l'infrastructure routière et du réseau d'eau potable ou d'assainissement et par conséquent la prolifération de vecteurs pathogènes et même la contamination de la nappe phréatique. Certes la pénurie est aujourd'hui moins pressante dans la plupart des communes d'Algérie grâce à la frénésie des forages, les connexions inter barrages et l'avènement des ressources hydriques non conventionnelles. La salinisation de la nappe par intrusion marine et sa contamination par les rejets divers, organiques et chimiques, la rend irréversiblement impropre à la consommation comme en témoigne déjà la fermeture de certains champs de captage. Dans les esprits, la pollution hydrique ou environnementale demeure à ce jour une menace lointaine ou étrangère alors que le processus une fois déclenché détruit des équilibres naturels et menace la santé humaine. Seul le secteur public de l'eau semble en avoir conscience. Ce qui est négligé ou ignoré aujourd'hui est fatalement différé au temps où nos enfants et petits enfants rallieront la course d'une planète en péril.

32 La même loi n04-20 régit la prévention des risques majeurs et la gestion des catastrophes en Algérie. Parmi les risques considérés majeurs, au nombre de dix (10), 
trois concernent directement la problématique des risques liés à l'eau. Il s'agit des inondations, la pollution hydrique et les risques climatiques. Concernant les deux premiers, rappelons qu'il s'agit-là d'aléas hydroclimatiques dérivés. Il est important d'en prendre note car l'objectif stratégique est d'en traiter en priorité les facteurs aggravants qui composent par ailleurs la vulnérabilité locale et non leurs effets. Or, si les causes des risques climatiques sont les aléas climatiques eux-mêmes, dans notre cas la pluviosité, les risques d'inondation et de pollution hydrique sont le résultat d'aléas hydroclimatiques naturels (les crues, l'intrusion marine,..). Autrement dit, la stratégie à adopter devrait consister à réduire les facteurs de vulnérabilité qui sont les véritables pourvoyeurs de risque en prévision des aléas naturels inévitables. L'article 6 annonce explicitement un objectif de «prise en charge des effets des risques majeurs sur les établissements humains"

Celles servant à la prévention de la pollution hydrique relèvent d'un plan particulier d'intervention dont l'objet est de prévoir le dispositif d'alerte et de maitriser les accidents (Art. 59). Les aléas climatiques, dont la sécheresse et les pluies importantes, font également l'objet d'un plan délimitant les zones exposées, les seuils d'alerte et toutes mesures de prévention « applicables lors de l'annonce des avis de pré-alerte ou d'alerte" (Art. 27). Les mesures de prévention elles-mêmes seraient adoptée a posteriori car « applicables lors de l'annonce des avis de pré-alerte ou d'alerte». Or, ces prescriptions relèvent davantage de la protection que de la prévention et davantage de l'atténuation que de l'adaptation.

La politique de management du risque semble par conséquent basée sur sa conservation et son transfert plutôt que sur la prévention. En ce sens où son programme ne prend pas suffisamment en charge les facteurs de survenance et de gravité et semble s'appuyer sur les opérations systématiques de secours et d'assurance pour le remboursement des frais d'endommagement (Art. 48). Dans ce cas, le management du risque se confond avec la gestion de catastrophe, en outre basée sur la planification des interventions par plan ORSEC pendant l'occurrence de l'aléa (Art.52) et certaines mesures structurelles par Plan Particulier d'Intervention consécutifs à la catastrophe. Celui-ci consiste à nommer une commission d'évaluation et de coordination chargée de répondre aux besoins urgents de la population en terme de soins, de nourriture, d'abri et de réparation des dommages (Art. 63). Si les réserves dites stratégiques qui permettent d'effectuer ces opérations peuvent bénéficier de dons de la part du gouvernement et de la population civile, la réparation des dommages se réduit à des aides financières que le bénéficiaire utilisera ou non à bon escient, car certains travaux de réhabilitation ou de restauration nécessitent une compétence technique spécifique.

Pour ce qui relève de la composante urbaine, les dispositifs de sécurisation stratégique ciblent principalement les infrastructures routières, les liaisons de communication et certains bâtiments à valeur stratégique ou patrimoniale (Chapitre 3, Section 1,2 et 3 ). Les mesures prévues auraient pour objectif d'assurer la continuité opérationnelle de ces ouvrages notamment en cas de séisme (Art. 43). Il peut être noté dans le texte l'usage impropre ainsi qu'une confusion de la terminologie du risque. A titre d'exemple, la sécurisation préventive semble relever davantage de la protection à court et moyen terme que de la prévention à long terme. D'autre part, les bâtiments sécurisés en priorité semblent identifiés en fonction de leur emplacement, leur mode de réalisation et leur ancienneté (Art.46). Ce qui nécessairement fait appel à une carte 
d'inondabilité par rapport à laquelle serait évalué leur niveau d'exposition, puis à une expertise technique et scientifique qui évaluerait leurs caractéristiques et l'intérêt historique. Il serait nécessaire de joindre à cette liste deux expertises pertinentes, l'une concernant le type d'activité et l'autre les mesures d'adaptation préalablement introduites.

Le texte de loi cité prévoit un plan général de prévention basé sur «l'observation permanente de l'évolution des aléas ou des risques, ainsi qu'une valorisation des informations enregistrées » (Art. 17). Par conséquent, la première mesure à prendre consisterait à lancer des études scientifiques permettant une meilleure connaissance de l'aléa et du risque potentiel qu'il porte. L'objectif stratégique serait donc l'élaboration de deux systèmes communiquant, l'un de veille et l'autre d'alerte servant respectivement la prévention et la gestion des secours en cas de catastrophe majeure.

Tels que fixés, les objectifs du système de veille seraient la connaissance de l'aléa, l'amélioration de sa prévisibilité et le déclenchement de l'alerte. Ce qui semble principalement servir la gestion de catastrophe et subsidiairement la prévention. Confirmé par l'Article 15, il fonde la prévention des risques majeurs sur des prescriptions générales et spécifiques, des dispositifs de sécurisation stratégique et «des dispositifs complémentaires de prévention». Alors que l'objectif intelligent, durable et prioritaire serait d'investir l'aléa par une meilleure connaissance du phénomène naturel qui le soutend et non de lutter contre ses effets après coup en établissant un rapport de forces inégales entre la nature et l'homme.

D'autre part, à l'échelle communale et pour ce qui concerne les risques liés à l'eau, la sécurisation isolée de certains districts ne peut être que relative, en tout cas aléatoire vu leur périmètre d'impact. La distribution hétérogène des enjeux, de la population, des activités et des infrastructures conforte en vérité le rôle de l'administration communale en tant qu'assemblée populaire et démocratique (APC) appelée à coopérer avec les forces humaines en présence et accorder un intérêt égal à tous ses habitants, quel que soit leur niveau de vie. Si privilège il devait y avoir, il irait indubitablement aux résidents des quartiers précaires.

39 Les procédures de sauvetage et de secours ne sont que des solutions provisoires qui diffèrent le risque à moyen terme. Or, pour devancer ce scénario tendanciel, la commune pourrait prendre appui sur ses forces internes et opportunités externes pour combler les insuffisances locales. Afin de transformer l'aléa naturel en opportunité et tirer profit de son énergie potentielle, la commune dispose déjà d'un remarquable arsenal d'outils politiques, juridiques et économiques lui permettant d'investir ses forces sociales et revenus économiques dans la revalorisation des zones humides et notamment les oueds et leurs berges, la réutilisation des eaux usées et déchets recyclables, qui en outre lui permettraient de créer des postes d'emploi. Les projets de résorption et réhabilitation de l'habitat précaire ou vétuste pourraient également constituer d'intéressantes opportunités de renouvellement urbain pris en charge par un outil de planification original permettant de conserver la spécificité sociale, environnementale et économique de la commune.

\section{CONCLUSION}

Aujourd'hui, les aléas climatiques font l'objet d'un suivi régulier de la part de l'Organisme National de Météorologie (ONM) à cause du risque potentiellement majeur 
qu'ils présentent. Or, si l'intérêt vital de l'eau n'est pas à démontrer, les enjeux de développement rappellent avec force la nécessité de les privilégier par rapport aux autres aléas naturels sans toutefois les en dissocier. A cette problématique s'ajoute depuis peu celle des changements climatiques qui a conduit à l'élaboration d'une stratégie nationale de lutte contre leurs effets probables (MATE, 2008). Celle-ci définit des orientations stratégiques et reste en attente de plans réglementaires et opérationnels.

41 C'est pourquoi, notre approche se veut critique, tant vis-à-vis des politiques et stratégies de développement urbain et de prévention, que des attitudes et comportements de la population face au risque et à l'aléa. Alors que pour la population qui l'encourt, le risque fait partie de la vie quotidienne et exige l'improvisation de réponses immédiates, provisoires s'il en est, pour les politiciens le programme d'action est fortement conditionné par la durée d'un mandat électoral et, de ce point de vue, devrait comprendre des réalisations tangibles fussent-elles provisoires. La différence d'attitude entre acteurs urbains sociaux et politiques vis-à-vis du risque est cependant une donne bien connue des scientifiques qui y reconnaissent les signes d'une pratique de gouvernance inadéquate sur le plan qualitatif et non celui des moyens, voire d'un développement général inégalement réparti. C'est à ce moment-là que l'information, l'éducation et l'instruction se justifient et s'imposent comme préalable au débat public sur le devenir souhaité de la commune, les objectifs de développement et le choix des processus à mettre en œuvre.

Bien que coûteuses, certaines solutions sont en effet avantageuses à terme et la motivation sociale ainsi que la volonté politique devraient contribuer à les inscrire parmi les priorités locales. C'est en investissant les services de la nature ainsi que le partenariat avec les organisations sociales non gouvernementales et l'université, en favorisant la synergie de leurs programmes et en rentabilisant les moyens humains disponibles, que la commune pourra, en toute légalité, se frayer un chemin vers l'indépendance d'action.

Les mesures techniques mises en œuvre semblent faillir en l'absence d'une gestion efficiente sur les plans quantitatif et qualitatif. Lorsque les inondations ne provoquent pas l'interruption des activités socioéconomiques quotidiennes, ou mettent en danger la vie des citoyens, ce sont la pénurie et les pollutions hydrique et environnementale qui contribuent à entretenir l'insalubrité du cadre de vie, par ailleurs vétuste ou précaire, et donc défavorisent la santé et l'hygiène publique. L'interprétation des résultats obtenus désigne par ailleurs tantôt des besoins à satisfaire et des problèmes à résoudre à l'échelle du bassin versant et tantôt des enjeux à traiter au niveau de la commune. Leur prise en charge exige le recours aux disciplines de planification et aménagement du territoire urbain, du paysage, des milieux naturels et de l'eau et nécessite la référence à des outils de planification stratégique, en cours d'élaboration. 


\section{BIBLIOGRAPHIE}

AROUA N., Facteurs de vulnérabilité et capacité de résilience du milieu urbain face aux risques hydroclimatiques dans la commune algéroise d'El Harrach, Thèse de doctorat en science, Ecole Polytechnique d'Architecture et d'urbanisme d'Alger, 2012.

AROUA N., Les ressources en eau dans le processus de planification urbaine durable. Cas de l'agglomération algéroise, Mémoire de Magister, Ecole Polytechnique d'Architecture et d'urbanisme d'Alger, 2005.

ADGER W. N., Vulnerability, in Global Environmental Change, Volume 16, Issue 3, August 2006, p. 268-281.

BROOKS N. et al., The determinants of vulnerability and adaptive capacity at the national level and the implication of adaptation, in Global Environmental Change, Part A, Volume 15, Issue 2, July 2005, p. 151-163.

ALWANG J. et al., Vulnerability: a view from different disciplines, The World Bank, Social protection discussion paper series $\mathrm{n}^{\circ} 0115$, June 2001, $42 \mathrm{p}$.

OULDAMER R., ALGERIA, Rapport national intermédiaire du suivi de la mise en œuvre du Cadre d'action de Hyōgo, Ministère de l'intérieur et des collectivités locales, décembre 2008.

FERNINI A., Evaluation de la vulnérabilité urbaine face aux risques majeurs naturels. Simulation partielle sur un quartier témoin Algérois, Mémoire de Magister, Ecole Polytechnique d'Architecture et d'Urbanisme (EPAU) d'Alger, Mai 2008.

\section{RÉSUMÉS}

En Algérie, en l'espace de quelques mois d'intervalle, la presse nationale peut rapporter des titres aussi contradictoires que les événements hydroclimatiques auxquels ils sont liés. La persistance de la sécheresse entre 1980 et 2000, avait conduit le pays à l'élaboration d'un Plan National de l'Eau (PNE) basé sur une déficience chronique de la ressource. Puis des pluies diluviennes se sont abattues sur l'ensemble du territoire provoquant de nombreuses inondations. La tragédie de Bab el Oued en Novembre 2001 en a été la plus meurtrière manifestation. Mais, alors que les autorités continuent à plaider le caractère naturel quoiqu'exceptionnel de tels événements, plusieurs localités à travers le pays tentent de faire face aux nombreux dégâts occasionnés avec les moyens du bord, c'est-à-dire dans le dénuement, parfois sans plan de secours préétabli. La gestion locale des risques liés à l'eau semble se restreindre aux secours d'urgence et au dépassement qui en résulte. Bien qu'appréhendé par la population et l'autorité locale, l'aléa naturel est encore vécu comme un hasard fatal contre lequel rien ne sert de se prémunir. Comment expliquer sans cela la prolifération de l'habitat précaire le long des oueds, voire dans leur lit, la marge étroite de la commune en terme de planification et de prise de décision pour ce qui relève de son propre territoire et sa population, enfin l'isolement traditionnel des acteurs publics et privés retranchés chacun derrière ses suffisances et insuffisances. Pour autant que la gestion locale $\mathrm{du}$ risque consiste à contrôler les événements dérivés des phénomènes hydroclimatiques, l'analyse multicritère des facteurs qui la déterminent, permet d'identifier certaines insuffisances structurelles dont l'impact à terme cultive le risque au lieu de le réduire. L'objectif de la présente communication est de tester cette hypothèse dans le cas de l'Algérie. 
In Algeria, in few months, the national press can report some news as contradictory as related hydroclimatic events. The persistence of drought between 1980 and 2000, led the country to develop a National Water Schema based on a chronic deficiency of the resource. Then heavy rains fell on the territory causing widespread flooding. The tragedy of Bab el Oued in November 2001 was the deadliest event. But while the authorities continue to plead the natural character of such events, many communities across the country are trying to cope with many damage with available means, that is to say in poverty, sometimes without previous emergency strategy. Local water related risk management seems to be restricted to emergency and resulting confusion. Although apprehended by the population and the local authority, the natural hazard is still considered as a fatal accident. How otherwise explain both the proliferation of squatter settlements along the river, even in its bed, and the municipality's narrow margin to plan and make decision related to its own territory and population, and the traditional isolation between public and private stakeholders. As far as the local risk management consists in controlling the events derived from the hydroclimatic phenomena, the multicriteria analysis of specific parameters may contribute to identify some structural deficiencies which impacts aggravate the risk rather than reduce it. Present paper aims at testing this hypothesis in the case of Algeria.

\section{INDEX}

Mots-clés : Algérie, analyse multicritère, gestion des risques liés à l'eau, résilience urbaine Keywords : Algeria, multicriteria analysis, urban resilience, water related risks management

\section{AUTEURS}

\section{NADJET AROUA}

Maître de conférences, Ecole Polytechnique d'Architecture et d'Urbanisme d'Alger (EPAU),

Email : arouanajet[CHEZ]yahoo.fr

\section{EWA BEREZOWSKA-AZZAG}

Professeur, Directeur de recherche, Ecole Polytechnique d'Architecture et d'Urbanisme d'Alger

(EPAU), Email : ewaazzag[CHEZ]yahoo.fr 